\title{
Investigation of the thermoacoustic process in the piping of metering equipment in a natural gas reducing station
}

\author{
S. Zarei ${ }^{1} \&$ H. Shokouhmand ${ }^{2}$ \\ ${ }^{1}$ University of Tehran, Iran \\ ${ }^{2}$ Schools of Mechanical Engineering, College of Engineering, \\ University of Tehran, Iran
}

\begin{abstract}
The main objective of this study was to investigate the untypical pipe vibrations in gas pressure reducing and flow metering stations in specific operating conditions (low flow) and its effect on the behavior of meters in the studied station. These vibrations are mainly caused by the thermoacoustic process in the area adjacent to the junction of two trains of regulators to heat exchangers. To simulate and study this phenomenon, a Rijke tube was used, which is an experimental model for this purpose. Then a mathematical model was developed to complete a theoretical analysis, and the main parameters of the system and its behaviour were investigated. The simulated model was then solved using the computational fluids dynamics techniques and was compared with the general behaviours in the thermoacoustic phenomenon. In the end, a number of solutions were presented to reduce the propagation of the vibrations. The solutions can be generalized from the Rijke tube model to the studied station. The main factors include the heat exchangers location and the amount of energy consumed in the stations.

Keywords: thermoacoustic oscillations, Rijke tube, pipe vibration, pressure reducing station.
\end{abstract}

\section{Introduction}

Observations and reports in a number of gas pressure reducing stations where the turbine flowmeter is installed upstream indicate unusual vibration with noise at low flow rates. For example, these observations have been made in a pressure reducing station, whose configuration and characteristics are presented in the 
following figure 1 . The station has a capacity of 10,000 cubic meters per hour and consists of two parallel trains. Each train consists of a filter separation, heater with common header, turbine flowmeter, pressure regulator, and safety valve. Vibrations usually happen at low flow rates when heat exchanger is in service and disappears again upon increasing the flow. Experience shows that vibrations excite with increase in the water temperature and, thereby, the heat transferred to gas. Vibrations in pipe system and structures cause significant error in flow measurements. It should be noted that, in a large number of pressure reducing stations, turbine flowmeters are located downstream (after regulator). Why this phenomenon does not happen in some stations is explained in the results section. Quantitative data and measurement of a sample station are needed to examine this problem more closely. Therefore, data from a similar sample station were used for simulations. The station is briefly described in the following [1]. In the end, results were generalized to different types of pressure reducing stations with different configurations.

The main cause of the phenomenon is the thermoacoustic instability between the heat transferred to the natural gas and the vibration of the gas column inside the pipes. Vibration in the structures and measurement fault in flowmeters occur due to this excitation. In this field, interesting cases such as combustion instabilities [2] and thermoacoustic engines [3] and lately thermoacoustic vibrations in some gas pressure reducing and metering stations [1] have been studied. The physical mechanism of the phenomenon and the various parameters and their effect on the vibration of system are described in the following. The simulated model, which is based on the Rijke tube, was then numerically solved.

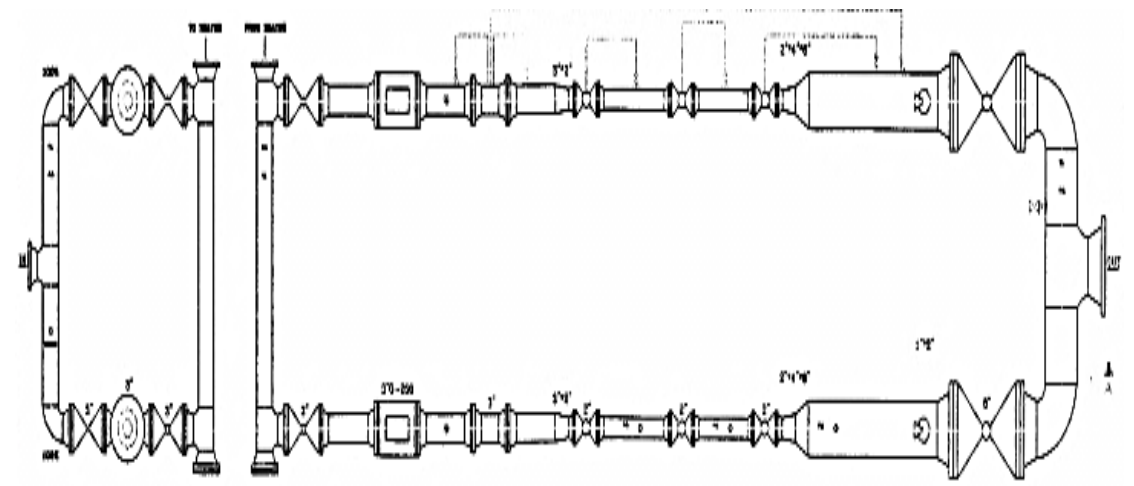

Figure 1: Schematic of a pressure reducing station with a capacity of 10,000 cubic meters per hour.

\section{Problem descriptions in a sample station}

Figure 2 presents an overview of the piping system and different vibration and pressure pulsation measurement points in the studied station [1]. 


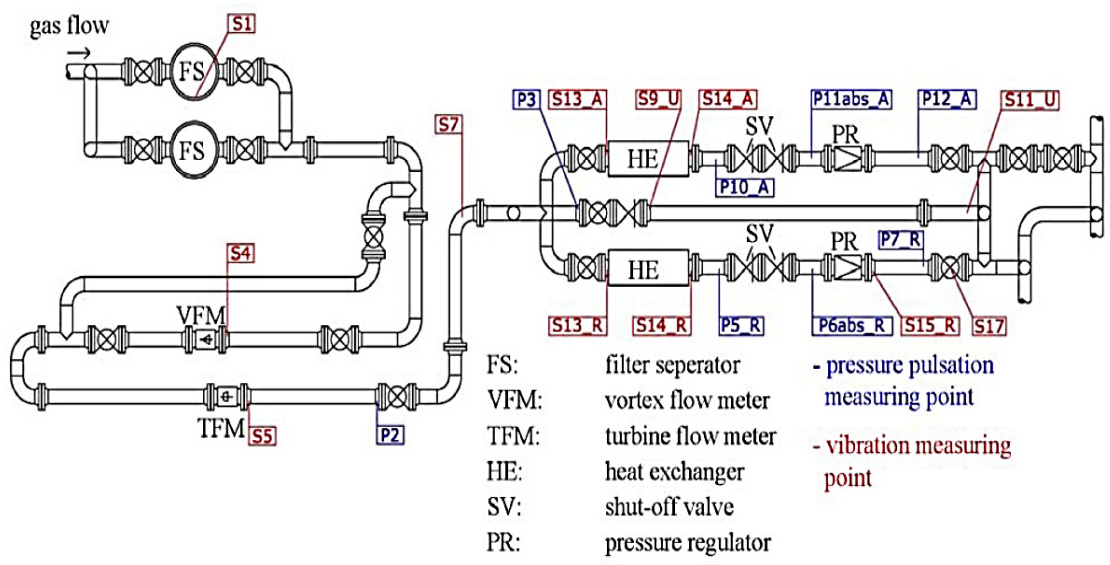

Figure 2: An overview of the piping system in the sample reducing station with different measuring points [1].

\section{Vibration analysis}

Thermoacoustic phenomena are classified as heat-induced spontaneous vibration that was investigated in a resonant tube by Rijke in late 19th century. Distribution of acoustic pressure and velocity is introduced as a function of the length of the tube [4] (figure 3).

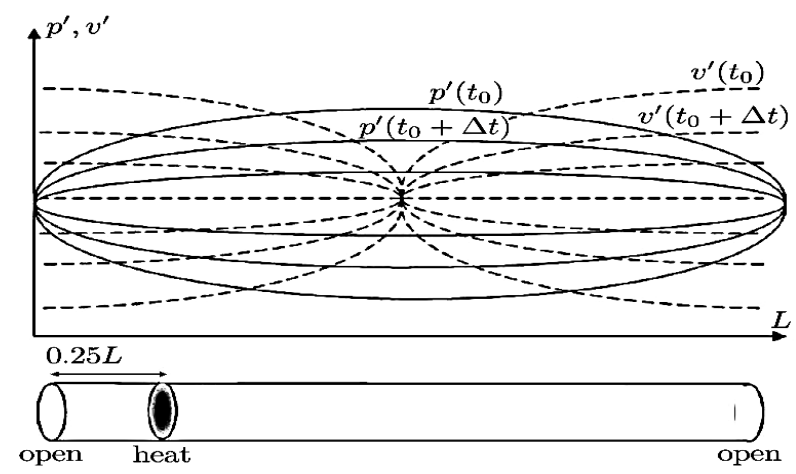

Figure 3: Resonance in the Rijke tube.

The criteria developed by Lord Rayleigh in 1878 [5] are still applied. These relations can be mathematically described by using the Rayleigh integral $I=\frac{1}{T} \int_{t}^{t+T} p^{\prime}(t) \cdot \dot{Q}^{\prime}(t) d t$. Here, $\dot{Q}^{\prime}(t), p^{\prime}(t)$ represent the fluctuation of heat release and acoustic pressure(pressure perturbation), with their product being integrated by a time period of oscillation $T$. depending on Rayleigh index $I$, 


\section{$I>0$ shows that the system is in resonance \\ $I<0$ shows that the system is being damped}

of a disturbance of average flow rates.

\subsection{Method of vibration analysis in Rijke tube}

The main goal of analysis is to calculate parameters such as pressure, velocity, temperature, energy, and density as a function of the main system variables such as time and space.

\subsection{Simplified model}

The model geometry is shown in Figure 4. We need a tube with both ends open with air flowing inside and a concentrated heat source. Common assumptions and analysis that have been used to reduce the complexity of the mathematical model are listed in the Matveev's research [6].

\subsection{Linear theory}

We disregard the two basic assumptions in 3.1.1 to improve the accuracy. This means that the effective power transferred from the heater to the air flow is calculated through heat transfer analysis, and a non-uniform temperature field is considered along the tube. The rest of the assumptions remain valid. Our starting point is the mechanical conservation equations in fluid such as mass, momentum and energy, and air is considered as an ideal gas. Pressure, density, and velocity in the airflow, and the added heat and volume source are in the form of sum of averages and oscillatory parts with small amplitude. for unit volume. The timedependent component of the volume source is zero. The steady component of pressure is assumed constant along the tube. Losses in the mean flow due to friction on walls are negligible. Acoustic disturbances occur in the Rijke tube in the presence of mean flow. Finally wave equation for pressure disturbance in the Rijke tube is derived [6].

$$
\frac{\partial^{2} p^{\prime}}{\partial t^{2}}-a^{2} \frac{\partial^{2} p^{\prime}}{\partial x^{2}}+\frac{a^{2}}{\rho_{\circ}} \frac{\partial \rho_{\circ}}{\partial x}+\frac{\partial p^{\prime}}{\partial x}=(\gamma-1) \frac{\partial \dot{Q}^{\prime}}{\partial x}+\rho_{\circ} a^{2} \frac{\partial \Omega^{\prime}}{\partial t}
$$

where, $\dot{Q}$ is the rate of heat transfer to the unit volume, and $\Omega$ is the volume source intensity. This equation is the basis for our analysis. Note that $a$ is the speed of sound, $\rho_{\circ}$ is density, and density gradient is a function of the longitudinal coordinate along the tubes.

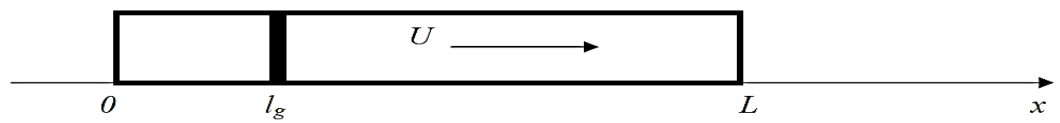

Figure 4: One-dimensional Rijke tube with a concentrated heat source. 


\subsection{Numerical analysis using computational fluid dynamics methods}

This study aimed to describe the coupling between the added heat and velocity and pressure oscillations in such environment. In the present model, flow field was assumed unsteady, compressible, and two-dimensional, where the added heat and acoustic interactions are combined. Results showed a relationship between pressure, velocity, and transferred heat fluctuations.

\subsubsection{Governing equations}

ANSYS Fluent v15 [7] was used for modeling. The entire process was performed by Finite Volume method. The governing equations consist of: conservation of mass equation, two-dimensional Navier-Stokes equation, energy equation and ideal gas standard equation.

Results are obtained by iteratively solving the four above equations in the software.

It should be noted that solving this problem consists of three steps. Since the problem is very time-consuming and unsteady, we can divide it into three steps to significantly reduce the time required to obtain the results. In the first step, to establish buoyancy conditions, a low gauge pressure of $0.5 \mathrm{~Pa}$ was applied to the tube inlet, and atmospheric pressure was applied to the end of the tube. This gives us a weak fluid flow in the tube. The temperature of the heat exchanger surface was considered $1200 \mathrm{~K}$ and the problem was once solved in the steady condition to reach complete convergence. The goal is the solutions of the steady case as initial conditions for the unsteady case. In the second step, we need to introduce a pressure disturbance in a short duration of time to make the model turbulent. In the third step, the unsteady problem is solved, which takes several hours. The pressure oscillations are obtained after thousands of time steps.

\subsubsection{Modeling using ANSYS WORKBENCH}

As mentioned earlier, our equivalent model is a Rijke tube with the length of $(\mathrm{L}=1 \mathrm{~m})$ and diameter of $(\mathrm{d}=0.06 \mathrm{~m})$. Air was considered as fluid, and heating element were considered at a distance of $25 \mathrm{~cm}$ from the left end. Flow direction was assumed left to right and the geometry of the problem was first modeled.

\section{Results and discussion}

\subsection{Description of Fluent output graphs}

Figure 5 shows that the location of heater is a key factor in the generation of oscillations in the Rijke tube. According to the thermoacoustic theory, when the heater is located at the middle of the tube, large amplitude oscillations do not occur. Thermoacoustic theory states that adding energy to system in a point with zero oscillation velocity (middle of the tube) does not create excitation.

According to figure 6, in this case, pressure does not have regular fluctuations like the case with the heater located at $\frac{X}{L}=\frac{1}{4}$. Figure 7 shows that increase in the source temperature and exceeding the critical value increases the acoustic wave's 
amplitude in the above variables, which is due to development of instability caused by heat input. According to figures 8 and 9, regular pressure and velocity oscillations have a phase difference of about 90 degrees.

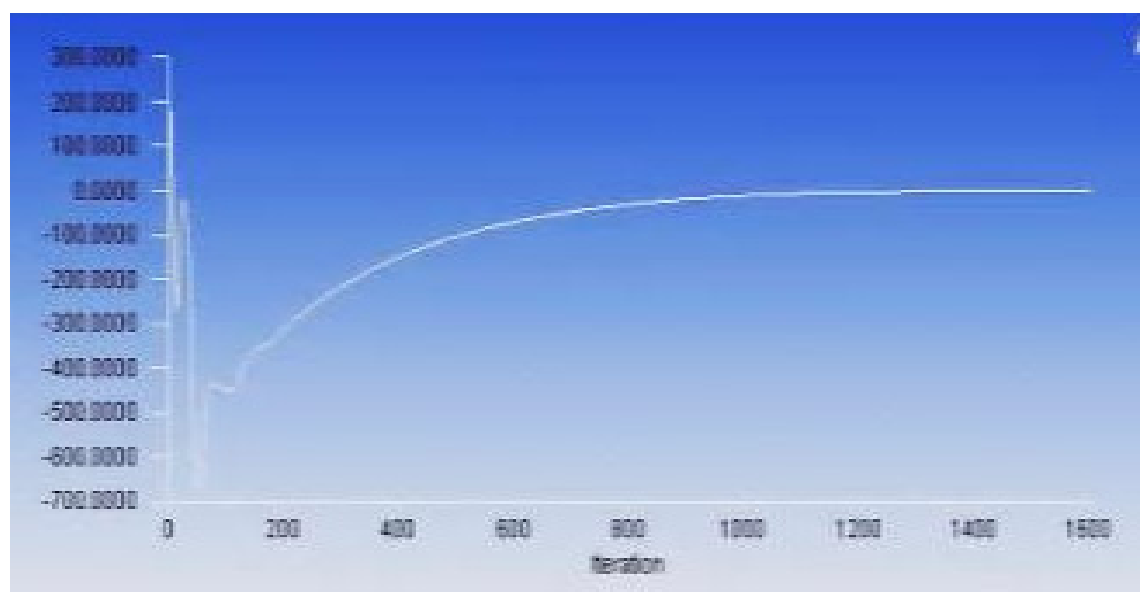

Figure 5: Pressure from the start of disturbance until regular fluctuations $T=1200 \mathrm{~K},\left(\frac{x}{L}=\frac{1}{2}\right)$.

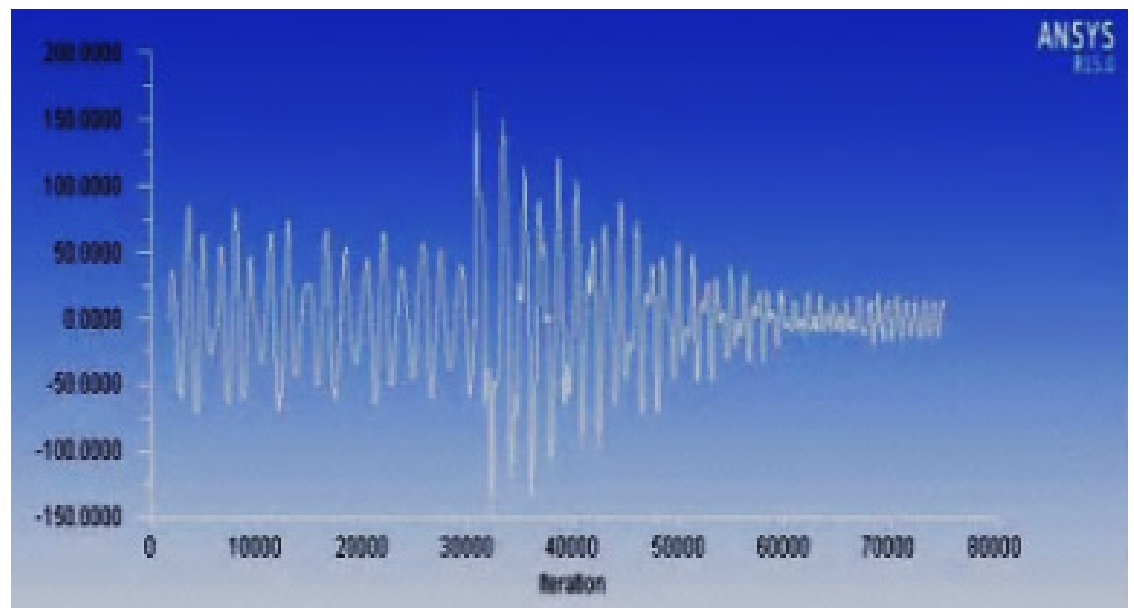

Figure 6: Pressure at the time of regular fluctuations at a point after the heater $T=1200 \mathrm{~K},\left(\frac{x}{L}=\frac{1}{2}\right)$. 


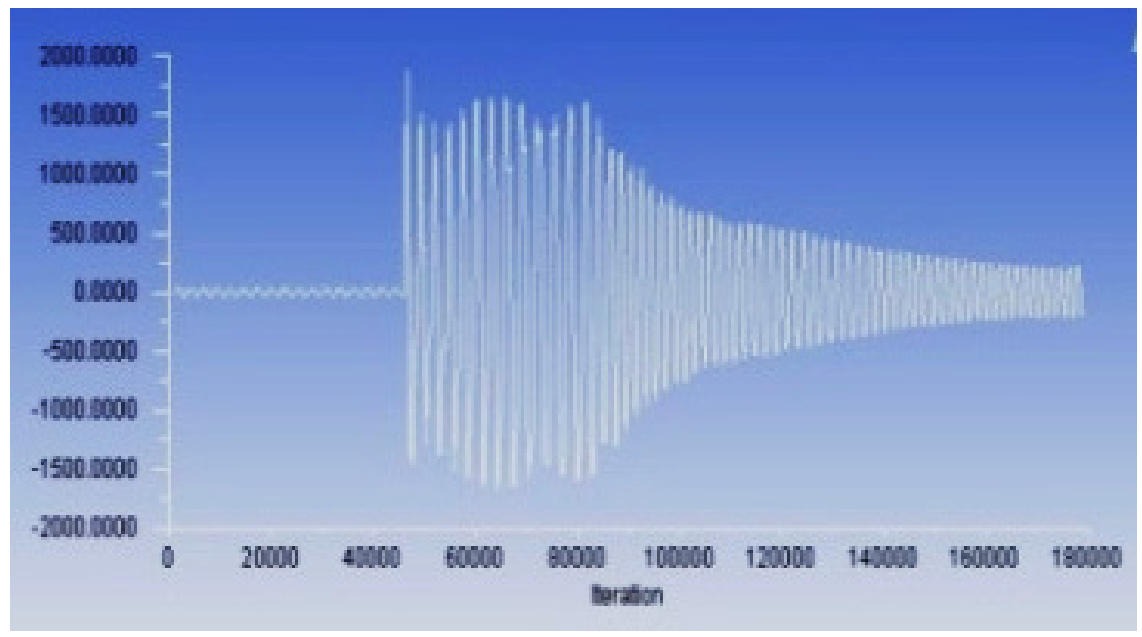

Figure 7: Pressure from the start of disturbance until regular fluctuations $T=3200 \mathrm{~K},\left(\frac{x}{L}=\frac{1}{4}\right)$.

\subsection{Comparison of output simulation diagrams with the sample station results}

In this section, to validate the data and diagrams obtained by the model simulations using Fluent, they were compared to numerical results of a sample gas pressure reducing station [1].

\subsubsection{Vibration after starting the heat exchanger}

If the heat exchanger starts, the base oscillation is intensified, and pressure waves grow exponentially after a time delay to converge to fixed value of amplitude and become balanced. Such behavior was also observed in gas pressure reducing and metering stations [1].

\subsubsection{System's response to a sudden heat input}

Thermoacoustic oscillations are caused by the phase difference between thermal and pressure oscillations in the area of heat input. In addition, the phase difference of $\frac{\pi}{2}$ between the velocity and pressure oscillations helps create fluctuations that are introduced to the system in the form of excitation waves. According to figures 8 and 9 the heat input was added to the system at $\frac{X}{L}=\frac{1}{4}$, which caused regular fluctuations in variables of pressure and velocity. If the heat is added to the system at $\frac{X}{L}=\frac{1}{2}$, according to figure 5 fluctuations will not occur very regularly or continue according to figure 6 . 


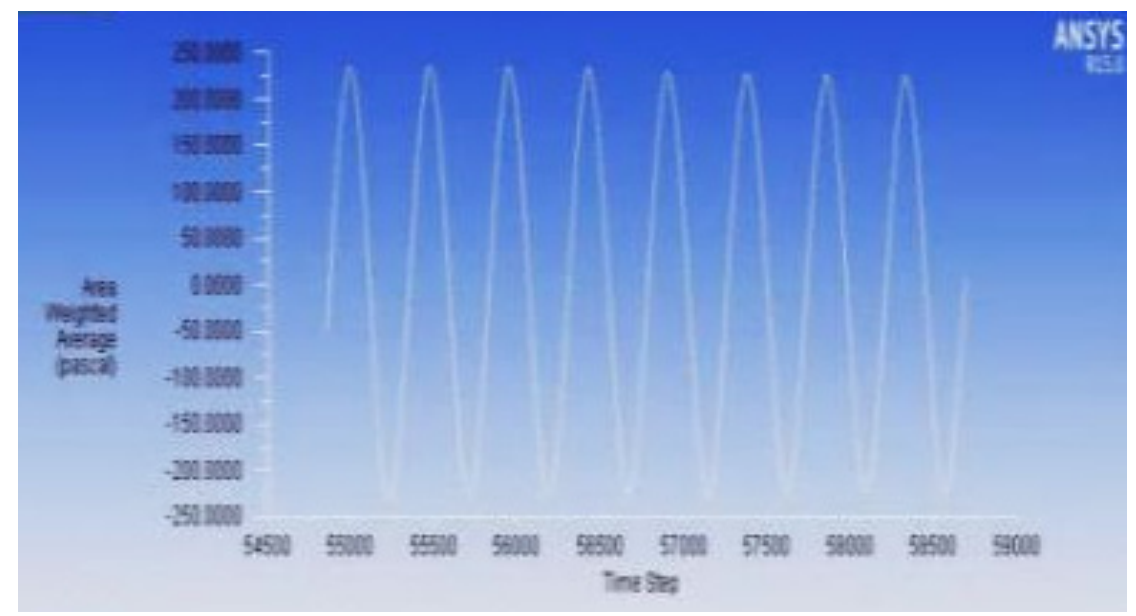

Figure 8: Pressure at the time of regular fluctuations at a point after the heater $\mathrm{T}=1200 \mathrm{Km}\left(\frac{x}{L}=\frac{1}{4}\right)$.

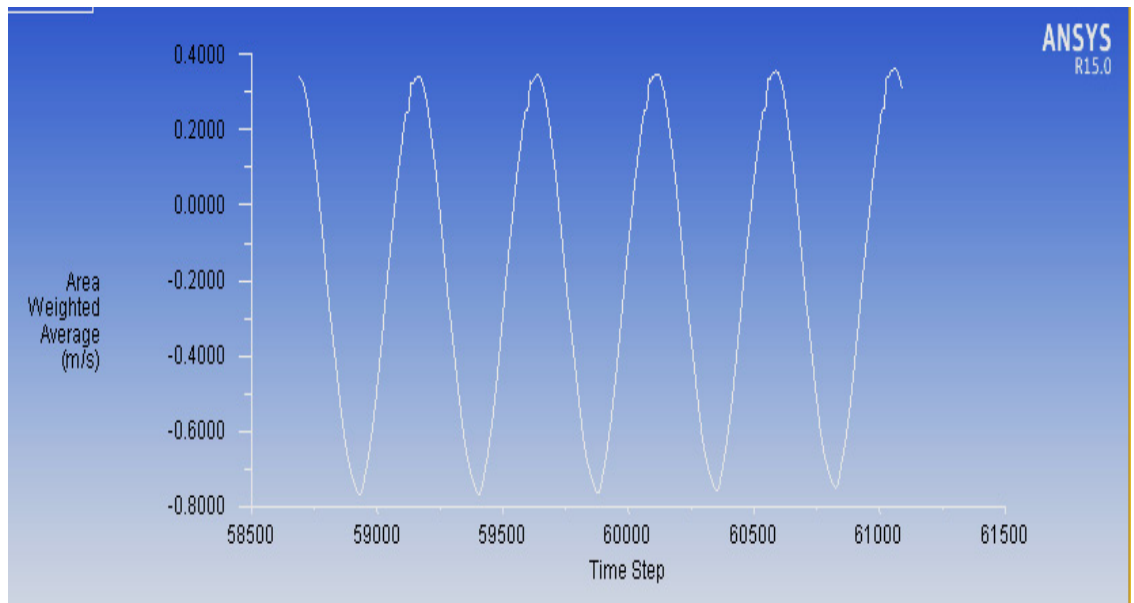

Figure 9: Velocity at the time of regular fluctuations at a point after the heater $\mathrm{T}=1200 \mathrm{~K},\left(\frac{x}{L}=\frac{1}{4}\right)$.

\subsubsection{Influence of heat exchanger temperature}

According to figure 7 and in comparison with figure 10, vibrations amplitude increased dramatically with increase in the heater (heat exchanger) temperature. In modellings in Fluent, the heat exchanger temperature was changed from $1200 \mathrm{~K}$ to $3200 \mathrm{~K}$. Other temperatures can also be added to this range in future research. 


\subsubsection{Heat exchanger location}

According to figure 5 when the heat exchanger (heater) is moved to $\frac{X}{L}=\frac{1}{2}$, fluctuations do not develop and do not turn into regular fluctuations as compared to when the heat exchanger is located at $\frac{X}{L}=\frac{1}{4}$. The evidence is in figure 10 . Also, by comparing figure 6 with figure 9 the reduced pressure amplitude especially after time step 55,000 is observed.

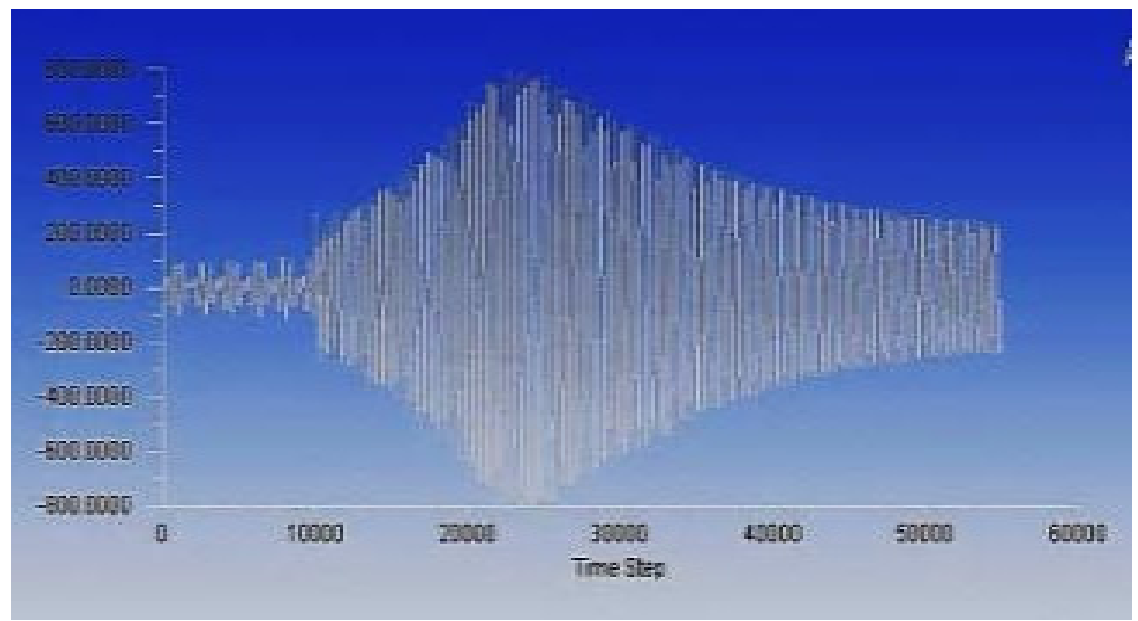

Figure 10: Pressure from the start of disturbance until regular fluctuations $T=1200 \mathrm{~K},\left(\frac{x}{L}=\frac{1}{4}\right)$.

\subsection{Solution criteria for preventing thermoacoustically induced vibration in gas pressure reducing and flow metering stations}

The calculation method should be applied to simulation of all gas pressure reducing and flow metering stations, particularly when new stations are designed with changes in operating conditions. For future simulation-based designs, the amount of consumed energy, heat exchanger location, and damping effects are main parameters in preventing thermoacoustic vibrations.

\subsubsection{Energy input}

Spread of thermoacoustic vibrations is strengthened especially at stations where the heat exchanger temperature is not adjusted or decrease based on minimum flow. A minimum allowable flow must be considered at stations where the control system does not turn the heat exchanger off. 


\subsubsection{Heat exchanger location}

At a given station, Rayleigh integral value can be calculated in pipes based on the geometry and the first natural frequency mode. Figure 11 shows the station control routes, which were described in the first section, in horizontal arrangement as the Rayleigh integral was calculated for the station. It was found that the in-service line connected to the heat exchanger is in the maximum range of Rayleigh integral and, therefore, intensifies the spread of thermoacoustic vibrations.

Excitation of vibrations can be prevented by placing the heat exchanger closer to the T-shaped piece or the feed line because it would be as if the heater is placed at $\frac{X}{L}=\frac{1}{4}$. In addition, depending on the existing stations, the stand-by line may be first equipped with a special connection by a driving motor or closed when the line is not in service. Consequently, the acoustic length of the tube is shortened, moving the maximum point of the Rayleigh integral towards the regulator. Therefore, thermoacoustic vibration amplitude is reduced or excitations are completely prevented. In internal gas pressure reducing stations, the input lines are usually connected to a common header before the flowmeters (figure 1). This header is connected to a set of indirect gas heaters, whose output is connected to another common header. Parallel lines, including a series of pressure regulators and the remaining components, are branched from that common header. Under such arrangement, considering the distance of heat exchanger from the T-shaped piece, the heater is not located at $\frac{X}{L}=\frac{1}{4}$ by default, reducing the spread of thermoacoustic vibrations.

\subsubsection{Damping}

Simulation results show that disturbance excitations do not last long in the presence of a damping in the system. Consequently, increased damping in the frequency range of the first natural vibration frequency of the pipe can prevent or reduce the associated acoustic vibrations. A gate can be installed in the vicinity of the T-shaped piece or at the inlet of heat exchanger. Or a damper can be installed where the fluctuation in velocity are high. As another alternative, a vertical arrangement can be provided for tube bundles in the heat exchangers with deflectors so as to increase the pressure loss. Compared to the horizontal arrangement of the heat exchanger, vibrations are distributed to the lowest desired damping. Another approach to prevent thermoacoustic vibrations is to prevent operation of the station in a flow less than a specified value (Reynolds number). Minimum allowable volumetric flow (Reynolds number) depends on the damping inside the respective pipe section and cannot be universal named. It should be noted that in the mentioned arrangement in the previous section and the common header before and after the heat exchanger, pressure drop in the pipe system actually increases and acts as a damper due to increased length and diameter of the header pipe. 


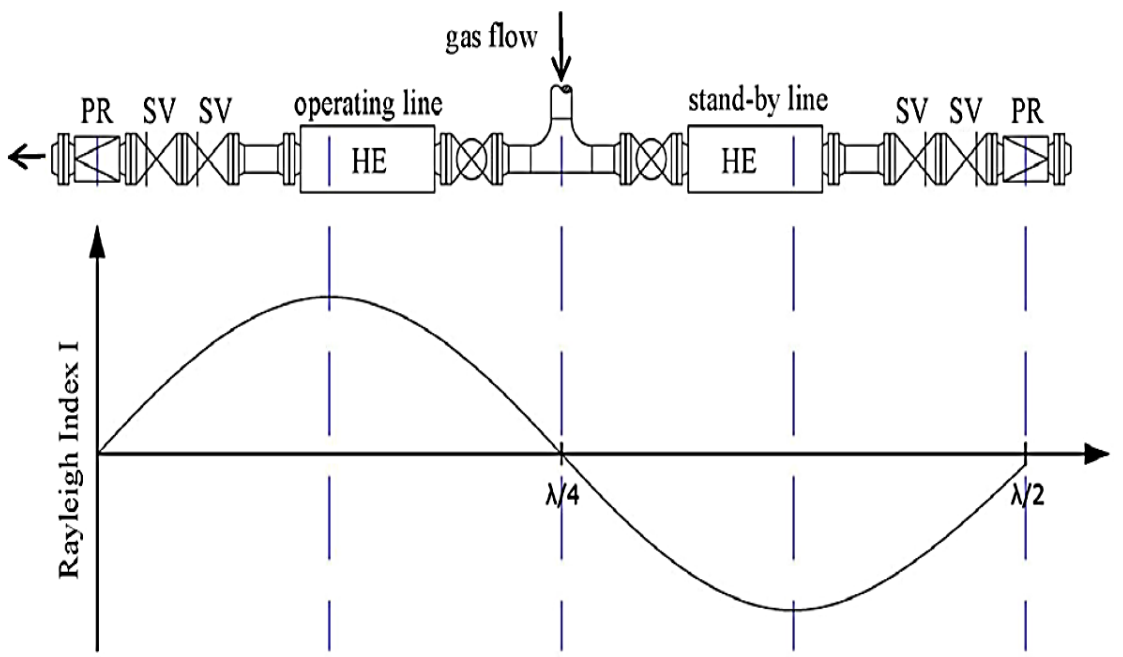

Figure 11: Rayleigh index calculation for the sample pressure reducing station (the right side is closed) [1].

\section{Conclusion}

Vibration and its influence on the behavior of flowmeters were discussed in a station. Such vibrations are mainly caused by the thermoacoustic process in the area adjacent to the junction of two trains of pressure regulator and the heat exchangers. Rijke tube is an empirical model that allows for the simulation of thermoacoustic oscillations. Numerical modeling of Rijke tube using computational fluid dynamic method helps determine the main effective factors in the physical mechanism of thermoacoustic vibration. Rayleigh integral law is approved to stimulate thermoacoustic fluctuation excitation. Finally, a number of approaches were proposed to prevent or reduce thermoacoustic vibrations, so that they can be generalized from the Rijke tube model to the station under consideration. The main effective factors include heat exchanger location, and the amount of energy used and damped in the station. For future research, spread of thermoacoustic vibrations must be prevented by the phase relationship between velocity oscillation and fluctuation in the input heat. Thermoacoustic effects must damp the pulsation in a kinematic manner by positive displacement devices using thermal electric regulator. Apart from theoretical considerations, experimental studies must also be performing. 


\section{References}

[1] Brümmer, A. \& Edlerherr, R. \& Lenz, J., Experimental and theoretical investigation of thermoacoustic oscillations in natural gas metering station. $15^{\text {th }}$ Flow Measurement Conference, 2010.

[2] Culick, F.E.C., Combustion instabilities in liquid-fuelled propulsion systems - an overview. AGARD-CP - 450, 1988.

[3] Swift G.W., Thermoacoustic Engines. Journal of the Acoustic Society of America, 84(1988), 4, 1148-1180.

[4] Remiorz L. \& Dykas L. \& Rulik S., Numerical modeling of thermoacoustic phenomenon as a contribution to the model of thermoacoustic engine. Task Quarterly 14 (2010), No. 3, 257-269.

[5] Rayleigh, Lord, The explanation of certain acoustic phenomena. Natural, 18: 319-321, 1878.

[6] Matveev, K. I. \& Culick, F. E. C., Experimental and mathematical modelling of thermoacoustic stabilities in a Rijke tube, $40^{\text {th }}$ Aerospace Meeting and Exhibit, Retro, NV, AIAA paper, 2002a.

[7] ANSYS, Inc. Release 15.0, "Fluent 15.0 Beta Features Manual". 